\title{
STUDIES OF THE INTERACTIONS OF IONIZING RADIATIONS WITH COMMUNICATIONS MATERIALS*
}

J. C. Ashley and M. W. Williams

Health and Safety Research Division

Oak Ridge National Laboratory

DOE/NBH -4002064

Oak Ridge, Tennessee 37830

DE84 002054

Air Force Interim Report on Work Conducted Under Project Orders RADC 81-10 (October 1980-September 1981) and RADC 82-09 (October 1981-September 1982)

\section{DISCLAIMER}

This report was prepared as an account of work sponsored by an agency of the United States Government. Neither the United States Government nor any agency thereof, nor any of their employees, makes any warranty, express or implied, or assumes any legal liability or responsibility for the accuracy, completeness, or usefulness of any information, apparatus, product, or process disclosed, or represents that its use would not infringe privately owned rights. Reference herein to any specific commercial product, process, or service by trade name, trademark, manufacturer, or otherwise does not necessarily constitute or imply its endorsement, recommendation, or favoring by the United States Government or any agency thereof. The views and opinions of authors expressed herein do not necessarily state or reflect those of the United States Government or any agency thereof.

\footnotetext{
* Research sponsored jointly by the Deputy for Electronic Technology, Air Force Systems Command, under Interagency Agreement DOE No. 40-226-70 and the Office of Health and Environmental Research, U.S. Department of Energy, under contract W-7405-eng-26 with the Union Carbide Corporation.
}

BV acceptance of this article, the publisher or recipient acknowledges the U.S. Government's right to retain a nonexclusive, royalty-free license in and to any copyright covering the article.

BISTRIBUTION OF THIS DOCUMEHT IS UHLLMTEED

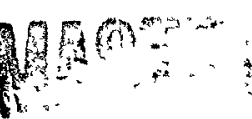




\section{DISCLAIMER}

This report was prepared as an account of work sponsored by an agency of the United States Government. Neither the United States Government nor any agency Thereof, nor any of their employees, makes any warranty, express or implied, or assumes any legal liability or responsibility for the accuracy, completeness, or usefulness of any information, apparatus, product, or process disclosed, or represents that its use would not infringe privately owned rights. Reference herein to any specific commercial product, process, or service by trade name, trademark, manufacturer, or otherwise does not necessarily constitute or imply its endorsement, recommendation, or favoring by the United States Government or any agency thereof. The views and opinions of authors expressed herein do not necessarily state or reflect those of the United States Government or any agency thereof. 


\section{DISCLAIMER}

Portions of this document may be illegible in electronic image products. Images are produced from the best available original document. 
STUDIES OF THE INTERACTIONS OF IONIZING RADIATIONS WITH COMMUNICATIONS MATERIALS

\section{Abstract}

This report summarizes experimental and theoretical studies designed to yield information on the interaction of ionizing radiations with various communications materials. In studies carried out during this 2-year period, we have developed various models for calculating the energy loss per unit length, or stopping power, and the inelastic mean free path for electrons in a material. We have included both organic materials such as polyethylene and inorganic materials such as $\mathrm{SiO}_{2}$. In each case, the calculated values of stopping power and mean free path have been compared with experimental values where available and, in the case of mean free paths for organic materials, with those predicted from our "universal" formula. In addition the various models for stopping power and mean free pàth have been compared with each other. Measurements have been made of electron attenuation lengths ( $\approx$ electron mean free paths) as a function of incident electron energy for electrons in amorphous carbon films, as a direct check on the values predicted by our universal formula for electron mean free paths in solid organic insulators. A theory has been developed to describe charged-particle track structure in nonmetallic solids, as the track evolves in time and space. Work has continued in the field of microdosimetry with the calculation of the energy densities deposited by high-energy photons as a function of depth in traversing a $\mathrm{SiO}_{2}$ slab sandwiched between two slabs of $\mathrm{Si}$. 
CONTENTS

PROGRAM SUMMARY ........................ . . 4

I. ENERGY LOSSES AND MEAN FREE PATHS OF ELECTRONS . . . . . . . 6

A. Silicon Dioxide............... 6

B. Summary of Mean Free Path Calculations . . . . . . 7

C. Polyethylene ................. 7

D. Condensed Organic Materials . . . . . . . . . 7

E. Additional Model Calculations . . . . . . . . . 8

II. ELECTRON ATTENUATION LENGTHS IN CARBON . . . . . . . . . . 14

III. CHARGED-PARTICLE TRACK STRUCTURE IN NON-METALLIC SOLIDS . . 18

IV. ELECTRON INTERACTIONS IN Si AND $\mathrm{SiO}_{2}$ IRRADIATED BY

PHOTONS ........................ 19

PUBLICATIONS AND PRESENTATIONS . . . . . . . . . . . . . 24 


\section{PROGRAM SUMMARY}

This report summarizes work done in an ongoing program under contract RAD 80007 for the periods October 1980 through September 1981 (Project Order RADC 81-10) and October 1981 through September 1982 (Project Order RADC 82-09). The objective is to gain information on the interaction of ionizing radiations with various communications materials. Thus, the intent is that work performed under this contract will increase our understanding of the basic mechanisms involved when photons, electrons, or other ionizing radiations interact with matter in its condensed phases. Emphasis is being placed on studying the interactions occurring in those materials which are currently used, or which are being considered for use, in electronics systems. Knowledge of quantities such as the cross sections for the various types of electron interactions in these materials enables predictions to be made concerning the performance of electronics systems in various situations, such as exposure to the results of a nuclear explosion. Our approach to this general problem is both theoretical and experimental. In our experimental program, we continue to make observations which can be related to photon and electron interactions with solids. The theoretical portions of the program included predictive calculations for a variety of interaction phenomena as well as interpretative studies in support of our experimental efforts. Results from the basic theoretical and experimental programs are employed in applied calculations with direct relevance to the performance of electronics systems including microsystems produced by VLSI technology.

In studies carried out during this 2-year period, we have developed various models for calculating the energy loss per unit length, or 
stopping power, and the inelastic mean free path for electrons in a material. We have included both organic materials such as polyethylene and inorganic materials such as $\mathrm{SiO}_{2}$. In each case, the calculated values of stopping power and mean free path have been compared with experimental values where available and, in the case of mean free paths for organic materials, with those predicted from our universal formula. In addition the various models for stopping power and mean free path have been compared with each other. Measurements have been made of electron attenuation lengths ( $\simeq$ electron mean free paths) as a function of incident electron energy for electrons in amorphous carbon films, as a direct check on the values predicted by our universal formula for electron mean free paths in solid organic insulators. A theory has been developed to describe charged-particle track structure in nonmetallic solids, as the track evolves in time and space. Work has continued in the field of microdosimetry with the calculation of the energy densities deposited by high energy photons as a function of depth in traversing a $\mathrm{SiO}_{2}$ slab sandwiched between two slabs of $\mathrm{Si}$.

The studies reported here were conducted by E. T. Arakawa, J. C. Ashley, R. N. Hamm, R. H. Ritchie, J. E. Turner, M. W. Williams, and H. A. Wright of the Health and Safety Research Division, ORNL, V. E. Anderson, Computer Sciences Division, ORNL, and T. A. Callcott and L. R. Painter, Department of Physics and Astronomy, University of Tennessee, Knoxville, Tennessee.

Finally, we gratefully acknowledge the continuing advice and support of J. N. Bradford of the Rome Air Development Center. 


\section{ENERGY LOSSES AND MEAN FREE PATHS OF ELECTRONS}

Our studies of the interaction of low-energy electrons with solids began over a decade ago and since then results from this work have been used in a wide variety of areas. It is particularly significant to note that our calculations of stopping powers of solids for electrons with energies $\leq 10 \mathrm{keV}$ form an important part of a new report prepared for submission to the International Commission on Radiation Units and Measurements (see M. J. Berger and S. M. Seltzer, "Stopping Powers and Ranges of Electrons and Positrons," NBSIR 82-2550, August 1982). During this reporting period, these studies have continued. The results are described below.

\section{A. Silicon Dioxide}

Earlier studies of electron interactions with $\mathrm{SiO}_{2}$ (RADC-TR-76-125, April 1976) have been, re-evaluated and refined for use in the microdosimetric studies undertaken in this reporting period. New theoretical results for stopping powers and mean free paths, and comparisons with experimental data, are described in detail in the following references:

1. "Interaction of Low-Energy Electrons with Silicon Dioxide," J. C. Ashley and V. E. Anderson, J. EZectron Spectrose. ReZat. Phenom. 24, 127-148 (1981).

2. "Energy Losses and Mean Free Paths of Electrons in Silicon Dioxide," J. C. Ashley and V. E. Anderson, IEEE Trans. Nucl. Sci. NS-28, $4132-4136$ (1981). 


\section{B. Summary of Mean Free Path Calculations}

Results for electron inelastic mean free paths in ten inorganic solids, some of which results were not readily available in the open literature, were collected and presented in a particularly simple form. Values of $k$ and $p$ were given for determining the mean free path $\lambda$ as a function of electron energy $E$ from the simple power-law expression $\lambda=k^{p}$ for values of $E$ from $200 \mathrm{eV}$ through $10 \mathrm{keV}$. The resulting table of $k-p$ values and comparisons of mean free paths with experimental data and with other theoretical results were given in the following paper: "Electron Inelastic Mean Free Paths in Several Solids for $200 \mathrm{eV} \leq \mathrm{E} \leq 10 \mathrm{keV}$,"

J. C. Ashley and C. J. Tung, Surf. Interface AnaZ. 4, 52-55 (1982).

\section{Polyethylene}

Previous results, derived using an insulator model, for the energy loss per unit path length and inelastic mean free path of an electron with an energy $\leq 10 \mathrm{keV}$ [Radiat. Res. 83, 1-18 (1980)] were modified by including an exchange correction, a sum-rule constraint, and a small relativistic correction. These new results were compared with the previous results, with tabulated values of stopping power based on Bethe theory, and with predictions from our universal formula for organic solids in the publication "Energy Losses and Inelastic Mean Free Paths of Low-Energy Electrons in Polyethylene," J. C. Ashley, Radiat. Res. 90, 433-436 (1982).

\section{Condensed Organic Materials}

A simple, "one-mode" approximation for the wavevector- and frequencydependent energy-loss function was employed to derive an expression for 
the electron inverse mean free path, $\lambda^{-1}$, including the effect of exchange between the incident electron and the electrons in the medium. The inverse mean free path in a specific material can then be obtained by averaging $\lambda^{-1}$ over the optical oscillator strength distribution which is proportional to $\omega \operatorname{Im}[-1 / \varepsilon(\omega)]$. For materials for which the optical oscillator strength distribution may be approximated by a single, Drudetype function, especially organic materials, the general results were cast in a particularly simple form. With four constants obtained from optical data on a given material, electron mean free paths in that material can be predicted for electron energies $\gtrsim 150 \mathrm{eV}$. An approximate expression was also deduced for estimating electron mean free paths in materials for which no optical data is available. Mean free paths determined from this approximate expression agreed well with those obtained from our earlier universal curve predictions. For polyethylene, excellent agreement between results from this model and those of a more detailed insulator model, as described in section $C$, gave additional support for this simple theoretical approach. Agreement between theoretical predictions and experimental measurements of electron mean free paths in organics, which in some cases is quite good, is on the whole less than satisfactory. These results are described in detail in: "Simple Model for Electron Inelastic Mean Free Paths: Application to Condensed Organic Materials," J. C. Ashley, Joumal of EZectron Spectroscopy and Related Phenomena (to be published).

\section{E. Additional Model Calculations}

We have continued to examine simple models for the response of solids to energy and momentum transfers from low-energy electrons 
( $\$ 10 \mathrm{keV})$ for prediction of inelastic mean free paths (IMFP) and energy losses per unit pathlength. Some of these results for the IMFP were discussed in section I-D of this report. Here we discuss predictions of stopping powers. We take

$$
\operatorname{Im}\left[(-1 / \varepsilon(q, \omega)]=\int_{0}^{\infty} \mathrm{d} \omega^{-} \omega^{-} \operatorname{Im}\left[-1 / \varepsilon\left(0, \omega^{-}\right)\right] \delta\left(\omega-\left(\omega^{-}+q^{2} / 2\right)\right) / \omega\right.
$$

for the energy-loss function which assumes a simple quadratic extension from the optical 1 imit $(q=0)$ into the energy and momentum transfer plane. The energy loss per unit pathlength, in atomic units, is calculated from

$$
S=\frac{2}{\pi v^{2}} \int d \omega \omega \int \frac{d q}{q} \operatorname{Im}[-1 / \varepsilon(q, \omega)] \equiv \int d \omega \omega \tau\left(\frac{v^{2}}{2}, \omega\right)
$$

where $\omega$ and $q$ for $\omega \geq 0$ are restricted to values for which $\omega \leq q(v-q / 2)$, and $v$ is the electron speed. For a fixed $w$, the values of $q$ lie in the range $q_{-} \leq q \leq q_{+}$where

$$
q_{ \pm} \equiv v \pm \sqrt{v^{2}-2 \omega}
$$

Equations (1)-(3) lead to

$$
\tau\left(\frac{v^{2}}{2}, \omega\right)=\frac{1}{\pi v^{2}} \int_{0}^{\infty} \mathrm{d} \omega^{\prime} \omega^{-} \operatorname{Im}\left[-1 / \varepsilon\left(0, \omega^{\prime}\right)\right] \mathrm{F}\left(\frac{v^{2}}{2}, \omega^{\prime}, \omega\right)
$$

where

$$
F\left(\frac{v^{2}}{2}, \omega^{-}, \omega\right) \equiv \theta\left(\omega-\frac{q_{-}^{2}}{2}-\omega^{-}\right) \theta\left(\omega^{-}-\omega+\frac{q_{+}^{2}}{2}\right) / \omega\left(\omega-\omega^{-}\right)
$$

and $\theta(x)=1$ for $x>0$ and $\theta(x)=0$ for $x<0$. We define an exchangecorrected differential inverse mean free path $\tau_{\text {exc }}$ by 


$$
\begin{aligned}
\tau_{\operatorname{exc}}\left(\frac{v^{2}}{2}, \omega\right) & \equiv \frac{1}{\pi v^{2}} \int_{0}^{\infty} d \omega^{-} \omega^{-} \operatorname{Im}\left[-1 / \varepsilon\left(0, \omega^{-}\right)\right]\left\{F\left(\frac{v^{2}}{2}, \omega^{-}, \omega\right)\right. \\
& \left.+F\left(\frac{v^{2}}{2}, \omega^{-}, \frac{v^{2}}{2}+\omega^{-}-\omega\right)-\left[F\left(\frac{v^{2}}{2}, \omega^{-}, \omega\right) F\left(\frac{v^{2}}{2}, \omega^{-}, \frac{v^{2}}{2}+\omega^{-}-\omega\right)\right]^{1 / 2}\right\},
\end{aligned}
$$

where the expression in \{\} reduces to a form proportional to the nonrelativistic M $\phi 1$ ler cross section when $\omega$ and $\frac{v^{2}}{2}-\omega$ are large compared

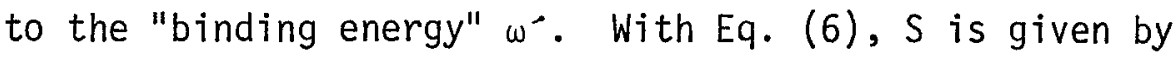

$$
\begin{aligned}
S=\frac{1}{\pi v^{2}} \int_{0}^{\frac{1}{2} \frac{v^{2}}{2}} d \omega^{\prime} \omega^{\prime} & \operatorname{Im}\left[-1 / \varepsilon\left(0, \omega^{-}\right)\right] \int_{\omega_{-}}^{\frac{1}{2}\left(\frac{v^{2}}{2}+\omega^{\prime}\right)} d \omega \omega\left[\omega\left(\omega-\omega^{\prime}\right)\right]^{-1} \\
& \left.+\left[\left(\frac{v^{2}}{2}+\omega^{-}-\omega\right)\left(\frac{v^{2}}{2}-\omega\right)\right]^{-1}-\left[\omega\left(\omega-\omega^{\prime}\right)\left(\frac{v^{2}}{2}+\omega^{-}-\omega\right)\left(\frac{v^{2}}{2}-\omega\right)\right]^{-1 / 2}\right\},
\end{aligned}
$$

where $\omega_{-} \equiv \frac{1}{2} \frac{v^{2}}{2}\left[1+\frac{2 w^{\prime}}{v^{2}}-\sqrt{1-\frac{4 w^{\prime}}{v^{2}}}\right]$.

The integrations over $\omega$ in Eq. (7) can be done analytically resulting in the following expression:

$$
\begin{aligned}
S=\frac{1}{\pi v^{2}} \int_{0}^{\frac{1}{2} \frac{v^{2}}{2}} \mathrm{~d} \omega^{\prime} \omega^{-} & \operatorname{Im}\left[-1 / \varepsilon\left(0, \omega^{-}\right)\right]\left\{\ln \frac{1-a}{1-a-s}+\ln \frac{1+a}{1+a+s}+\frac{1}{a} \ln \left[\frac{(1+a)(1-a+s)}{(1-a)(1+a+s)}\right]\right. \\
& \left.-7\left(\sin ^{-1} \frac{s}{1-a}, \frac{1-a}{1+a}\right)+\frac{1}{2} \ln [(1+a / 2-\sqrt{a(1+a / 4)}) / a]\right\} .
\end{aligned}
$$

In this expression $a \equiv 2 \omega^{-} / v^{2}, s \equiv(1-2 a)^{1 / 2}$, and $\mathcal{Y}$ is an elliptic integral of the first kind defined by 


$$
\exists(\phi, k)=\int_{0}^{\sin \phi} d x\left[\left(1-x^{2}\right)\left(1-k^{2} x^{2}\right)\right]^{-1 / 2}
$$

Given a set of optical data over the required energy range, the integral over $\omega^{-}$in Eq. (8) could be done numerically to obtain S. However, a simple expression can be obtained by expanding the analytic functions resulting from the $w$ integration in powers of $\omega^{1} / v^{2}$. If we assume $v^{2} / 4$ is large compared to the value of $\omega^{-}$for which $\omega^{-} \operatorname{Im}\left[-1 / \varepsilon\left(0, \omega^{-}\right)\right]$ is largest, then only the first few terms in the expansion should be sufficient to approximate $S$.

Through second order terms in $\omega^{-} / v^{2}$, we find

$$
\begin{aligned}
S \approx \frac{2}{\pi v^{2}} & \int_{0}^{\frac{1}{2} \frac{v^{2}}{2}} d \omega^{\prime} \omega^{\prime} \operatorname{Im}\left[-1 / \varepsilon\left(0, \omega^{\prime}\right)\right]\left[\ln \frac{v^{2}}{\omega^{\prime}} \sqrt{\frac{e}{8}}-\frac{3}{2} \frac{\omega^{\prime}}{v^{2}}-\frac{1}{2} \frac{\omega^{\prime}}{v^{2}} \ln \frac{2 v^{2}}{\omega^{\prime}}\right. \\
& \left.+\sqrt{2}\left(\frac{\omega^{\prime}}{v^{2}}\right)^{3 / 2}-\frac{1}{4}\left(\frac{\omega^{\prime}}{v^{2}}\right)^{2} \ln \frac{2 v^{2}}{\omega^{\prime}}-\frac{31}{12}\left(\frac{\omega^{\prime}}{v^{2}}\right)^{2}\right] .
\end{aligned}
$$

From this form, in the large $v 1$ imit, we obviously recover the nonrelativistic Bethe formula

$$
S_{B, N R}=\frac{\Omega_{p}^{2}}{v^{2}} \ln \frac{v^{2}}{I} \sqrt{\frac{e}{8}},
$$

where $\Omega_{p}^{2}=4 \pi n_{0} Z, n_{0}$ is the number of atoms or molecules per unit volume with $Z$ electrons per atom or molecule and $I$ is the mean excitation energy defined by

$$
I=\int_{0}^{\infty} \mathrm{d} \omega^{-} \omega^{-} \operatorname{Im}\left[-1 / \varepsilon\left(0, \omega^{-}\right)\right] \ln \omega^{-} / \int_{0}^{\infty} \mathrm{d} \omega^{-} \omega^{-} \operatorname{Im}\left[-1 / \varepsilon\left(0, \omega^{-}\right)\right] .
$$


The stopping power has been evaluated from Eq. (10) for polyethylene using our optical data [Radiat. Res. $\underline{83}, 1-18$ (1980)], including carbon K-shel1 contributions, with the results shown in Table I ("this result").

Table I. Stopping Power of Polyethylene

\begin{tabular}{|c|c|c|c|}
\hline \multirow[b]{2}{*}{$E(e V)$} & \multicolumn{3}{|c|}{ Stopping Power in $\mathrm{eV} / \AA$} \\
\hline & This Result & "R.R." & "Bethe" \\
\hline 80 & 2.68 & 3.00 & 2.12 \\
\hline 100 & 2.92 & 3.14 & 2.67 \\
\hline 150 & 2.92 & 3.05 & 2.85 \\
\hline 200 & 2.72 & 2.82 & 2.73 \\
\hline 300 & 2.30 & 2.37 & 2.38 \\
\hline 400 & 1.97 & 2.05 & 2.08 \\
\hline 600 & 1.56 & 1.64 & 1.66 \\
\hline 800 & 1.31 & 7.38 & 1.40 \\
\hline 1000 & 1.74 & 1.20 & 1.21 \\
\hline 1500 & 0.882 & 0.917 & 0.917 \\
\hline 2000 & 0.724 & 0.757 & 0.747 \\
\hline 4000 & 0.437 & 0.454 & 0.445 \\
\hline 6000 & 0.320 & 0.331 & 0.324 \\
\hline 8000 & 0.255 & 0.263 & 0.258 \\
\hline 10000 & 0.213 & 0.221 & 0.216 \\
\hline
\end{tabular}

These results are in good agreement with our recent results, column "R.R.," Table I, obtained using an insulator model to describe the valence electrons and generalized oscillator strengths for K-shell 
ionization (see section C). Surprisingly, both sets of results agree with the Bethe-theory predictions, Eq. (11), (Column "Bethe," Table I), to within a few percent down to $200 \mathrm{eV}$. Part of the difference in the $S$ values in Table I between "this result" and "R.R." is due to a difference in $\mathrm{I}$ in the two cases. From the energy-loss function obtained from optical data, $I=62 \mathrm{eV}$. The insulator-model results ("R.R.") involved a fit of a sum of terms to $\operatorname{Im}[\varepsilon(0, \omega)]$. This function, plus the Kramers-Kronig relation between the real and imaginary parts of $\varepsilon$, were used to determine the valence-electron contribution to $\operatorname{Im}[-1 / \varepsilon(0, \omega)]$. This, plus the K-shell contribution as determined from generalized oscillator strengths, resulted in $\mathrm{I}=58 \mathrm{eV}$. This slightly smaller I corresponds to slightly larger values of $S$ than $I=62 \mathrm{eV}, \mathrm{e.g} .$, a $7 \%$ difference in I gives about a 1.5\% difference in $S$ at $8000 \mathrm{eV}$ according to Eq. (11).

The advantage of this model calculation is that a reasonably simple function, when integrated over the optical energy loss function, yields $S$ from Eq. (10). The disadvantage is that the optical energy-loss function is not available for all materials of interest. A more general problem is that almost no measurements are available of stopping powers for electrons with energies less than $10 \mathrm{keV}$ as a check on the theoretical descriptions. 


\section{I. ELECTRON ATTENUATION LENGTHS IN CARBON}

Measurements have been made of electron attenuation lengths as a function of incident electron energy over the energy range from 100 to $3000 \mathrm{eV}$ for electrons in amorphous carbon films. These measurements were undertaken as a direct check on the values predicted by our universal expression for the mean free path of electrons in solid organic insulators [Radiat. Res. 81, 364-373 (1980); IEEE Trans. NucZ. Sci. NS-27, 1454-1458 (1980)].

The attenuation lengths were obtained from electron transmission measurements through self-supporting carbon films. The films were prepared by the arc evaporation of high purity carbon electrodes in vacuum. Carbon was deposited onto glass slides, which had been spincoated with a dilute soap solution, at a rate of approximately $5 \AA$ /sec. With the soap acting as a parting agent, the carbon films were floated off the glass slides onto distilled water, and then picked up on 829line-per-inch $\mathrm{Ni}$ mesh. The films, which had thicknesses in the range from 60 to $200 \AA$, were examined using an optical microscope to locate continuous segments which might be suitable for the electron transmission measurements. Since electrons could be transmitted freely through holes too small to be resolved optically, only those segments for which the electron transmission became unmeasurable at low incident electron energies were used in the measurement of electron attenuation lengths.

Electrons, accelerated by an electron gun, were directed at the sample, and the electrons transmitted in a relatively large solid angle about the forward beam direction were collected. In order to discriminate against the majority of the electrons which had been scattered inelastically, 
the detector incorporated a retarding potential grid which was set at ten volts less than the electron gun accelerating potential. For each incident electron energy, the electron attenuation length, L, associated with inelastic scattering was then obtained from the electron transmittance, $T$, using the relationship

$$
T=e^{-t / L}=\frac{I-I_{B G}}{I_{0}-I_{B G}},
$$

where $t$ is the sample thickness, $I_{0}$ is the incident electron intensity, $I$ is the transmitted electron intensity, and $I_{B G}$ is the background intensity.

The experiments were performed in an ion-pumped, ultra-high vacuum chamber. To reduce the effect of magnetic fields on the electron beam, a mu-metal shield enclosed the sample region. Holes were cut in the shield for the electron gun, the detector and target assemblies, and for an observation window: Great care had to be used in the initial pumpdown to avoid rupturing the fragile films. An oil-free mechanical pump, pumping through a sma11 tube, was used first, followed by sorption and ion pumps. The ultimate vacuum was about $10^{-8}$ Torr, increasing to $5 \times 10^{-8}$ Torr when the electron gun was in operation.

The thicknesses of the films were determined from the measured transmittance of He-Ne laser photons, using a transmittance versus thickness curve which had been calibrated using a quartz crystal thickness monitor. The thicknesses were 76, 98, 103, and $163 \AA$ with experimental uncertainties in these numbers of $\pm 20 \%$.

The electron attenuation lengths obtained for the four carbon films used in this study are shown in Fig. II-1 as a function of electron 


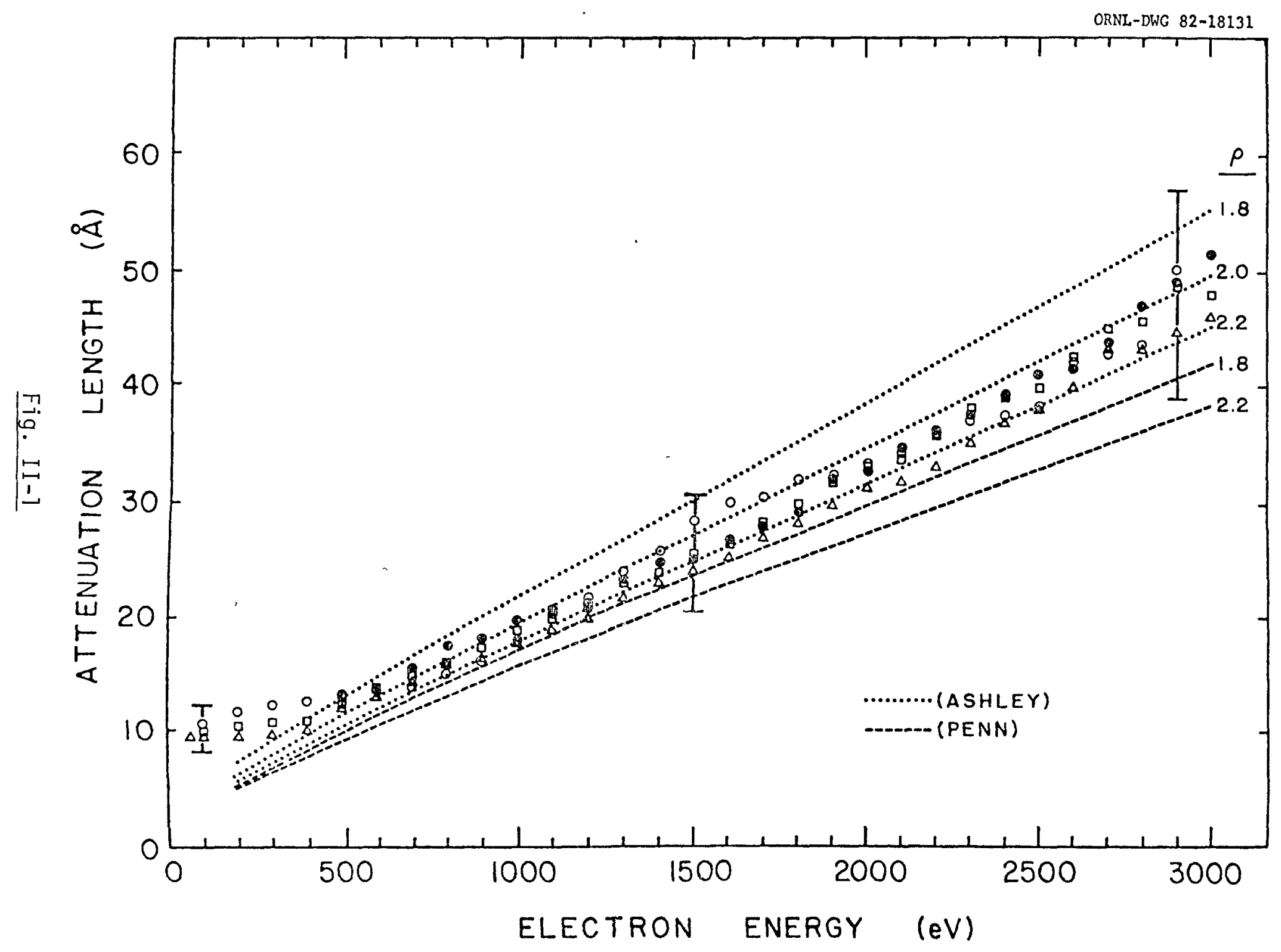


energy. Representative error bars are shown, and these result primarily from the $20 \%$ uncertainty in the film thicknesses. The dotted curves are calculated using our "universal" expression for electron mean free paths in solid organic insulators [IEEE Trans. Nuct. Sei. NS-27, 1454-1458 (1980)] for carbon film densities of $1.8,2.0$, and $2.2 \mathrm{~g} \cdot \mathrm{cm}^{-3}$. The dashed curves are calculated using Penn's equation [J. Electron. Spectrosc. Relat. Phenom. 9, 29-40 (1976)] based on a free electron gas model.

To make a valid comparison between experiment and theory it is necessary to know the density of the amorphous carbon films used in the electron transmission measurements. A preliminary determination of this density gave $2.1 \mathrm{~g} \cdot \mathrm{cm}^{-3}$. Assuming this value of density to be correct, examination of Fig. II-1 shows that the experimental values of electron attenuation length are in good agreement with the predictions of electron mean free path obtained from our universal curve from $\sim 500$ to $3000 \mathrm{eV}$. Below $2500 \mathrm{eV}$, the experimental values of electron attenuation length are larger than predicted from the universal curve. Furthermore, the observed dependence of electron attenuation length on energy is not that which is predicted. One reason for the differences between experiment and theory for incident electron energies $\leqslant 500 \mathrm{eV}$ may be that some inelastically scattered electrons may be included in the measured transmitted intensity, I. Alternatively, the universal curve may not apply in this energy region. Further studies are proposed to clarify the questions raised by this study. 
III. CHARGED-PARTICLE TRACK STRUCTURE IN NON-METALLIC SOLIDS

Our core plasma model of the expanding, partially compensated positive ion track created when a densely ionizing charged particle proceeds through matter has been generalized so that the collective effects are included a priori. The time evolution of delta rays that are generated in the primary physical stage of the energy deposition process is followed by means of an age-type approximation that includes the selfCoulomb forces of the delta-ray distribution as well as the Coulomb attraction of the positive ion column. On a slower time scale the ion column expansion is described self-consistently in a hydrodynamical framework and the atomic recoil distribution generated in the expansion is modeled on a multi-group diffusion approximation. Calculations with this scheme will furnish a complete description of the evolution in time and space of the energy distribution of atomic recoils and the ultimate conversion of this kinetic energy into thermal energy in a detailed space-time representation. Monte Carlo checks of these calculations are planned. Some results of this work have been published in: "A Core Plasma Model of Charged Particle Track Formation in Insulators," R. H. Ritchie and C. Claussen, NucZ. Instrum. Methods 198, 133-138 (1982) 
IV. ELECTRON INTERACTIONS IN $\mathrm{Si}$ and $\mathrm{SiO}_{2}$ IRRADIATED BY PHOTONS

Preliminary calculations were carried out of the relative energy densities deposited as a function of depth by monoenergetic $x$-ray photons incident on a $0.5-\mu \mathrm{m}$ slab of $\mathrm{SiO}_{2}$ sandwiched between two slabs of $\mathrm{Si}$. The slabs were infinite in lateral extent and the photons entered as a parallel beam perpendicular to the planes of the $\mathrm{Si}-\mathrm{SiO}_{2}-\mathrm{Si}$ interfaces. The front Si layer was 3-um thick and the back layer was essentially infinitely thick. Photon energies of $8.04 \mathrm{keV}, 17.4 \mathrm{keV}$, and $60.0 \mathrm{keV}$ were used. The first two energies are, respectively, those of the $k_{\alpha}$ characteristic $x$ rays of $\mathrm{Cu}$ and Mo. The photoelectric effect dominates in these two cases. The energy $60.0 \mathrm{keV}$ was chosen as an example in which photoelectric absorption and Compton scattering are about equally probable both in the case of $\mathrm{Si}$ and of $\mathrm{SiO}_{2}$.

Figure IV-I shows the energy deposited per unit volume (arbitrary units) in the array as a function of depth for irradiation by $8.04 \mathrm{keV}$ photons incident from the left. The $\mathrm{SiO}_{2}$ slice is located between the depths of $3.0 \mu \mathrm{m}$ and $3.5 \mu \mathrm{m}$. Rather than selecting the photon interaction sites at random, we let them occur at uniform intervals in the Si and $\mathrm{SiO}_{2}$, the linear density being adjusted in the ratio of the total macroscopic cross sections for photon interaction. Since our primary purpose is to study the effects of the interface between the two materials, it is not necessary to have photon interactions at all depths in the array. To calculate the data for Fig. IV-1, we allowed photons to interact only between the depths of about $2.5 \mu \mathrm{m}$ and $4.0 \mu \mathrm{m}$, indicated by the vertical arrows on the abscissa in Fig. IV-1. These depths were sufficiently far from the $\mathrm{SiO}_{2}$ layer to establish electronic equilibrium in the $\mathrm{Si}$ on 


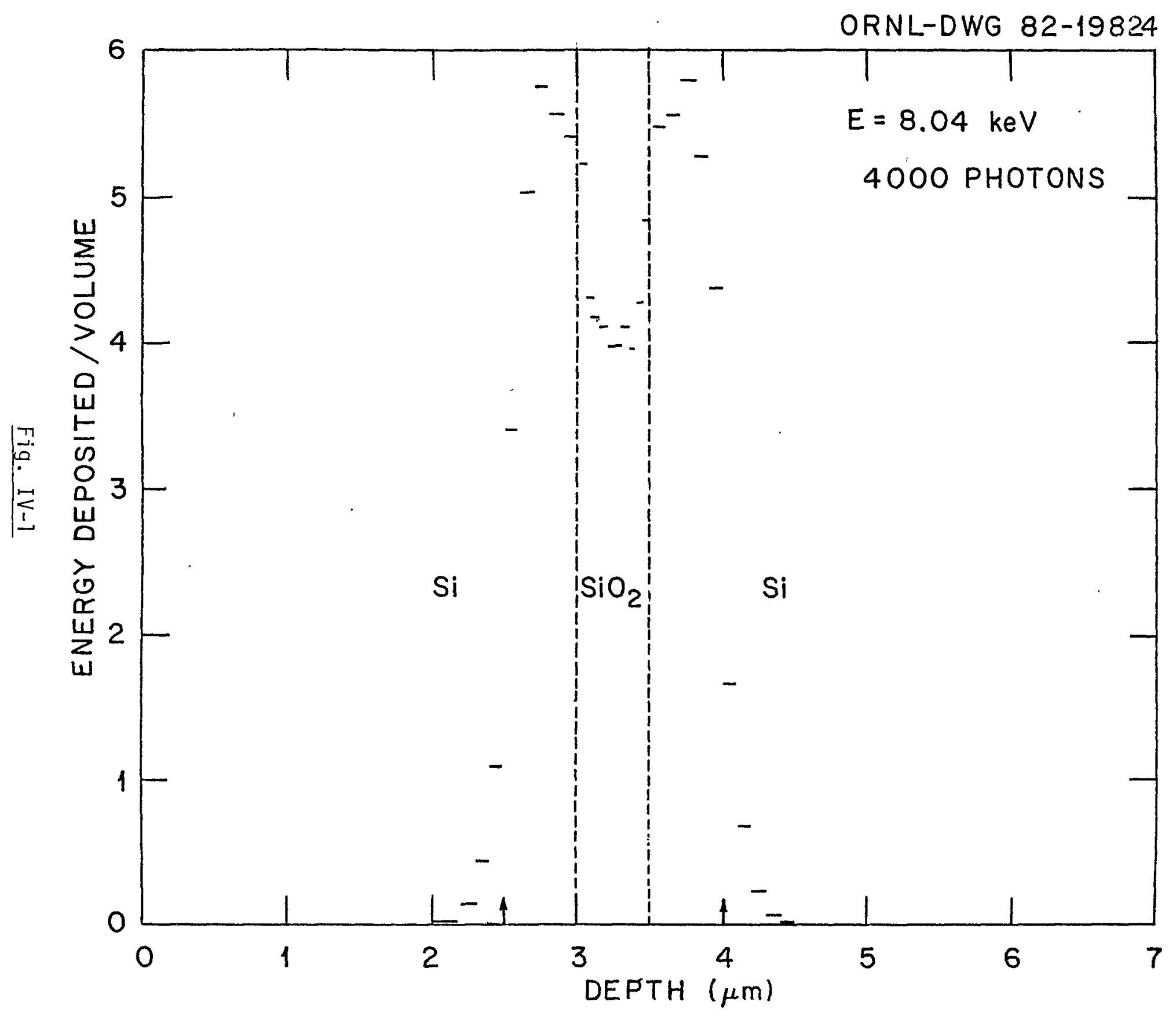


both sides of it. The results show that the energy deposited per unit volume drops as one goes from the $\mathrm{Si}$ into the $\mathrm{SiO}_{2}$ layer in the direction the photons are traveling. The energy deposited per unit volume then rises in going from the $\mathrm{SiO}_{2}$ into the $\mathrm{Si}$ on the back side. These results show a gradient in energy deposition across the $\mathrm{Si}-\mathrm{SiO}_{2}$ interface. A total of 4000 photon interactions were used in obtaining the data for Fig. IV-1.

Figure IV-2 shows preliminary results for the $17.4 \mathrm{keV} x$ rays. In this case 1800 photon interactions were used. An energy deposition gradient in the $\mathrm{SiO}_{2}$ layer is also seen here.

Figure IV-3 shows the raw data calculated for the 60-keV photons. The statistics are poor, as only 450 interactions were used spread over the interval from 0.1 to $6.4-\mu \mathrm{m}$ depth. If one combines the data for the $\mathrm{SiO}_{2}$ in Fig. IV-3 in intervals of $0.2 \mu \mathrm{m}$ instead of the $0.1 \mu \mathrm{m}$ shown, there is evidence of a gradient similar to those found at the two lower photon energies.

Based on these figures, final decisions will be made on the geometrical configurations and photon energies of most interest. Final computer runs will then be made with a sufficient number of photon interactions to assure satisfactory statistical resolution in the results. 


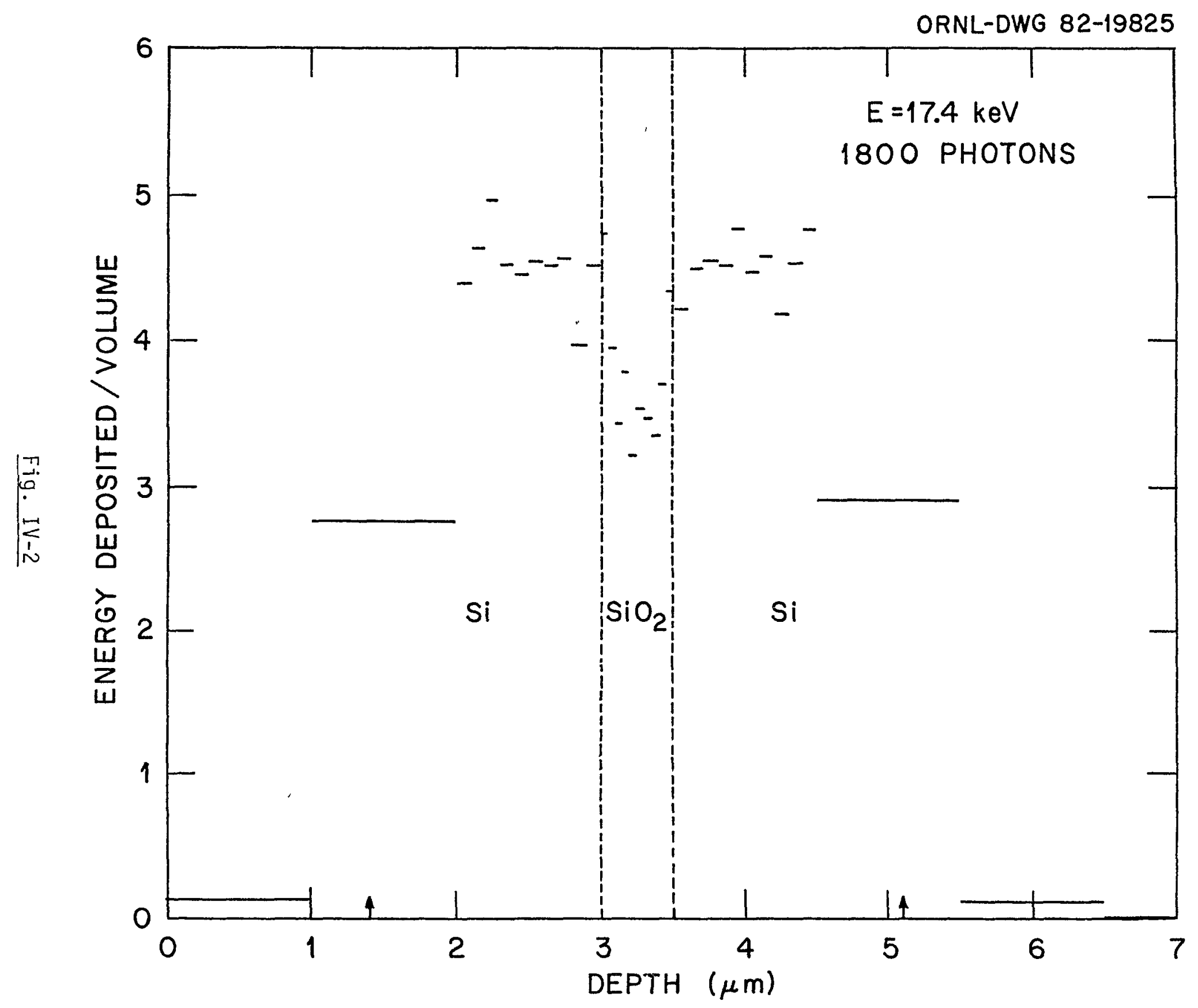




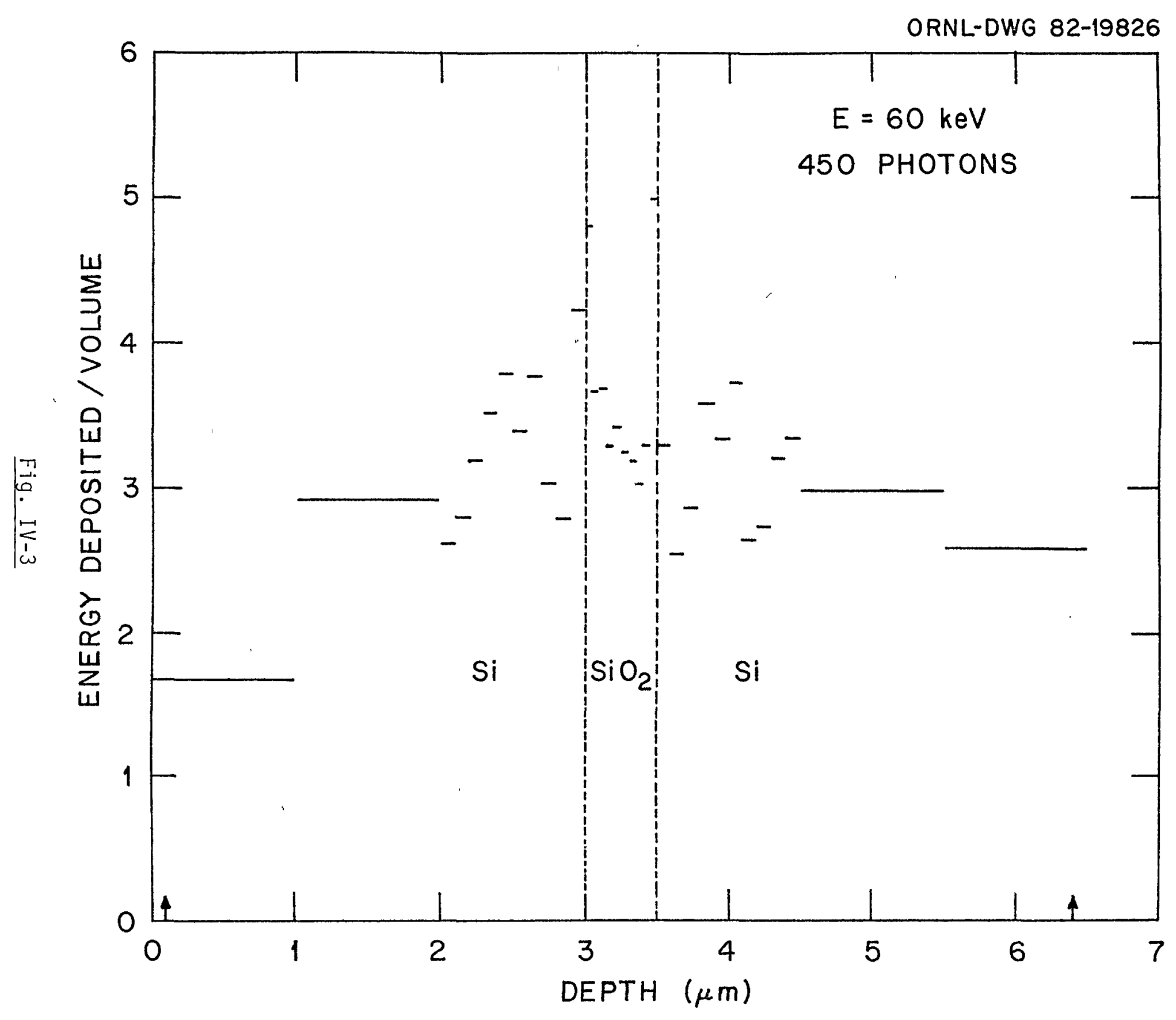




\section{PUBLICATIONS AND PRESENTATIONS}

During this reporting period several papers were published, or prepared for publication, and presentations were given at conferences which describe work related to this project and for which partial support by the Air Force was acknowledged. A list of these items follows.

\section{Publications}

1. "Inelastic Interactions of Low-Energy Electrons with Organic Solids: Simple Formulae for Mean Free Paths and Stopping Powers," J. C. Ashley, IEEE Trans. NucZ. Sci. NS-27, 1454-1458 (1980).

2. "Studies of the Interactions of Ionizing Radiations with Communications Materials," M. W. Williams and J. C. Ashley, Air Force Final Technical Report for October 1978 through September 1980, RADC-TR-80-348 (November 1980).

3. "Calculated Distance Distributions of Energy Transfer Events in Irradiated Liquid Water," R. N. Hamm, J. E. Turner, H. A. Wright, and R. H. Ritchie, in Proceedings of the Seventh Symposium on Microdosimetry, edited by J. Booz, H. G. Ebert, and H. D. Hartfiel (Harwood Academic Publishers, London, 1981), pp. 717-726.

4. "Interface Effects on Dose Distributions in Irradiated Media," H. A. Wright, R. N. Hamm, and J. E. Turner, in Proceedings of the Seventh Symposium on Microdosimetry, edited by J. Booz, H. G. Ebert, and H. D. Hartfiel (Harwood Academic Publishers, London, 1981), pp. 293-302.

5. "The Optical Properties of Kapton: Measurement and Applications," E. T. Arakawa, M. W. Williams, J. C. Ashley, and L. R. Painter, J. Appz. Phys. 52, 3579-3582 (1981). 
6. "Interaction of Low-Energy Electrons with Silicon Dioxide,"

J. C. Ashley and V. E. Anderson, J. EZectron Spectrose. ReZat. Phenom. 24, 127-148 (1987).

7. "Energy Losses and Mean Free Paths of Electrons in Silicon Dioxide," J. C. Ashley and V. E. Anderson, IEEE Trans. NucZ. Sci. NS-28, $4132-4136(1981)$.

8. "Energy Spectra of Heavy Fragments in the Interaction of Protons with Communications Materials," R. N. Hamm, M. L. Rustgi, H. A. Wright, and J. E. Turner, IEEE Trans. Nucl. Sci. NS-28, 4004-4006 (1981).

9. "Energy Losses and Mean Free Paths of Low-Energy Electrons in Polyethylene," J. C. Ashley, Radiat. Res. 90, 433-436 (1982).

10. "User's Manual for PHOEL-3, A Monte Carlo Computer Code for Calculating Initial Energies of Electrons and Positrons Produced in Water Irradiated by Photons with Energies up to $1 \mathrm{GeV}, "$ A. S. Todo, G. Hiromoto, J. E. Turner, R. N. Hamm, and H. A. Wright, ORNL/TM-8259 (June 1982).

11. "Electron Inelastic Mean Free Paths in Several Solids for $200 \mathrm{eV}$ S $E \leq 10 \mathrm{keV}, " \mathrm{~J}$. C. Ashley and C. J. Tung, Surf. Interface Anal. $\underline{4}$, 52-55 (1982).

12. "Calculated Initial Energies of Electrons in $\mathrm{Si}$ and $\mathrm{SiO}_{2}$ Irradiated by Photons with Energies Up to $2 \mathrm{MeV}, " \mathrm{~A}$. S. Todo, J. E. Turner, R. N. Hamm, and H. A. Wright, Nuclear Instmuments and Methods (to be published).

13. "Monte Carlo Calculations of Initial Energies of Electrons in Water Irradiated by Photons with Energies Up to $1 \mathrm{GeV}, "$ A. S. Todo, G. Hiromoto, J. E. Turner, R. N. Hamm, and H. A. Wright, Health Physics (to be published). 
14. "A Core Plasma Model of Charged Particle Track Formation in Insulators," R. H. Ritchie and C. Claussen, Nucl. Instrum. Methods 198, 133-138 (1982).

15. "Energy Losses by Swift Charged Particles in the Bulk and at the Surface of Condensed Matter," R. H. Ritchie, Nucl. Instrum. Methods 198, $81-91$ (1982).

16. "Collective Aspects of Charged-Particle Track Structure in Non-Meta11ic Solids," R. H. Ritchie, in Proceedings of the Eighth Symposium on Microdosimetry, September 1982 (to be published).

17. "Simple Model for Electron Inelastic Mean Free Paths: Application to Condensed Organic Materials," J. C. Ashley, Joumal of EZectron Spectroscopy and Related Phenomena (to be published).

18. "Physical and Chemical Evolution of an Electron Track in Liquid Water," H. A. Wright, J. E. Turner, R. N. Hamm, R. H. Ritchie, J. L. Magee, and A. Chatterjee, in Proceedings of the Eighth Symposium on Microdosimetry, September 1982 (to be published).

\section{Presentations}

1. "Energy Spectra of Heavy Fragments in the Interaction of Protons with Communications Materials," R. N. Hamm, M. L. Rustgi, H. A. Wright, and J. E. Turner, IEEE Annual Conference on Nuclear and Space Radiation Effects, University of Washington, Seattle, Washington, July 21-24, 1981.

2. "Energy Losses and Mean Free Paths of Electrons in Silicon Dioxide," J. C. Ashley and V. E. Anderson, IEEE Annual Conference on Nuclear and Space Radiation Effects, University of Washington, Seattle, Washington, Ju1y 21-24, 1981. 
3. "Physical and Chemical Evolution of an Electron Track in Liquid Water," H. A. Wright, J. E. Turner, R. N. Hamm, R. H. Ritchie, J. L. Magee, and A. Chatterjee, Eighth Symposium on Microdosimetry, Jülich, West Germany, September 27-0ctober 1, 1982.

4. "Collective Aspects of Charged-Particle Track Structure in Non-Metallic Solids," R. H. Ritchie, Eighth Symposium on Microdosimetry, Jülich, West Germany, September 27-October 1, 1982. 